\title{
The Sequential Trauma Score - A New Instrument for the Sequential Mortality Prediction in Major Trauma*
}

\author{
S. Huber-Wagner ${ }^{1}$, J. Stegmaier ${ }^{1}$, P. Mathonia ${ }^{1}$, T. Paffrath ${ }^{2}$, E. Euler ${ }^{1}$, W. Mutschler ${ }^{1}$, K.-G. Kanz ${ }^{1}$, R. Lefering 3 , \\ on behalf of the Working Group on Polytrauma (NIS) of the German Trauma Society (DGU)**
}

\author{
${ }^{1}$ Munich University Department of Trauma Surgery - Campus Innenstadt, Ludwig-Maximilians-University, Munich, Germany \\ ${ }^{2}$ Department of Trauma and Orthopedic Surgery, University of Witten/Herdecke, Cologne-Merheim Medical Center, Cologne, Germany \\ ${ }^{3}$ IFOM - Institute for Research in Operative Medicine, \\ University of Witten/Herdecke, Cologne-Merheim Medical Center, Cologne, Germany
}

\begin{abstract}
Background: There are several well established scores for the assessment of the prognosis of major trauma patients that all have in common that they can be calculated at the earliest during intensive care unit stay. We intended to develop a sequential trauma score (STS) that allows prognosis at several early stages based on the information that is available at a particular time. Study Design: In a retrospective, multicenter study using data derived from the Trauma Registry of the German Trauma Society (2002-2006), we identified the most relevant prognostic factors from the patients basic data (P), prehospital phase (A), early (B1), and late (B2) trauma room phase. Univariate and logistic regression models as well as score quality criteria and the explanatory power have been calculated.

Results: A total of 2,354 patients with complete data were identified. From the patients basic data $(\mathrm{P})$, logistic regression showed that age was a significant predictor of survival $\left(\mathrm{AUC}_{\text {model }} \mathrm{P}\right.$, area under the curve $=0.63)$. Logistic regression of the prehospital data (A) showed that blood pressure, pulse rate, Glasgow coma scale (GCS), and anisocoria were significant predictors $\left(\mathrm{AUC}_{\text {model A }}=0.76 ; \mathrm{AUC}_{\text {model } \mathrm{P}+\mathrm{A}}=0.82\right)$. Logistic regression of the early trauma room phase (B1) showed that peripheral oxygen saturation, GCS, anisocoria, base excess, and thromboplastin time to be significant predictors of survival $\left(\mathrm{AUC}_{\text {model B1 }}=0.78\right.$; $\left.\mathrm{AUC}_{\text {model P }+\mathrm{A}+\mathrm{B} 1}=0.85\right)$. Multivariate analysis of the late trauma room phase (B2) detected cardiac massage, abbreviated injury score (AIS) of the head $\geq 3$, the maximum AIS, the need for transfusion or massive blood transfusion, to be the most important predictors $\left(\mathrm{AUC}_{\text {model B2 }}=0.84 ; \mathrm{AUC}_{\text {final model } \mathrm{P}+\mathrm{A}+\mathrm{B} 1+\mathrm{B} 2}=0.90\right)$. The explanatory power - a tool for the assessment of the relative impact of each segment to mortality is $25 \%$ for $\mathrm{P}, 7 \%$ for $\mathrm{A}, 17 \%$ for $\mathrm{B} 1$ and $51 \%$ for $\mathrm{B} 2$. A spreadsheet for the easy calculation of the sequential trauma score is available at: www.sequential-trauma-score.com
\end{abstract}

\footnotetext{
* The paper has been presented orally at the annual meeting of the German Trauma Society (DGU) in Berlin 22.10.25.10.2008.
}

Conclusions: This score is the first sequential, dynamic score to provide a prognosis for patients with blunt major trauma at several points in time. With every additional piece of information the precision increases. The medical team has a simple, useful tool to identify patients at high risk and to predict the prognosis of an individual patient with major trauma very early, quickly and precisely.

Key words: Major trauma; outcome; prognosis; scoring; score; severely injured patients; polytrauma; dynamic score; ISS; TRISS; RISC; STS

\section{INTRODUCTION}

Trauma is one of today's most relevant health issues. In 2005 for example, a total of 173,753 deaths in the US were classified as injury-related. With a rate of 196.8 deaths/100,000 population it was the leading cause of death up to the age of 54 in 2005 [1]. The incidence has constantly increased over past years [1].

Characterization of the severity of injury is crucial for the scientific study of trauma, triage, classification of patients, quality management and the assessment of prognosis (prediction of mortality of an individual patient) [2-4]. In the scoring of the severity of trauma, mortality is the outcome that is of the most interest. Scores try to summarize and integrate a patient's condition into a one-dimensional value depending on many independent factors. More than 50 score systems have been published for the classification of injured patients in emergency or intensive care medicine. This large number indicates that prediction of outcome is and never will be perfect because severity of injury is complex and difficult to quantify [3].

There are several well established scores for the assessment of the prognosis of patients with major trauma [2, 4]. In 1974 the injury severity score (ISS) was introduced, [5] based on an anatomic classification, the abbreviated injury scale (AIS) introduced in 1971 [6]. It can be stated that the ISS is one of the most commonly used trauma score $[2,4]$.

The trauma score (TS) of 1981 and its further development, the revised trauma score (RTS, 1989) included physiologic variables such as the Glasgow coma 
scale (GCS), blood pressure, and respiratory rate [7, 8]. Since it was recognized that both the anatomic and the physiologic variables contain important prognostic information Boyd et al. [9] introduced the trauma and injury severity score (TRISS) in 1987 that combined both aspects. TRISS was widely adopted and as well as ISS, is one of the most commonly used trauma scores [2, 4]. In 1996 Osler et al. [10] introduced the "international classification of disease-9 based injury severity score" (ICISS). Its assessment of the pattern of injury is not based on the AIS but on the International Classification of Diseases, 9th revision [11]. In 1997 the new injury severity score (NISS) was introduced, that integrates several injuries of one anatomic region, compared with the ISS [12]. In 1990 Champion et al. [13] published a further attempt to improve the prediction of outcome of trauma; the "A severity characterization of trauma" score (ASCOT) in which the assessment of the injury pattern was modified compared with the TRISS. In 2001 Rixen et al. [14] introduced a score based on the Trauma Registry of the German Trauma Society (DGU), which for the first time evaluated and integrated the prognostic power of the base excess and the thromboplastin time $[15,16]$.

To increase the applicability and the predictive accuracy, the Institute for Research in Operative Medicine (IFOM) at the University of Witten/Herdecke in Cologne, Germany, recently developed a new score, the Revised Injury Severity Classification score (RISC). This score is based on the data of the trauma registry of the DGU. The RISC comprises more variables than the TRISS. If there are missing values, other variables are substituted, for example, if partial thromboplastin time is missing thromboplastin time is substituted. RISC-adjusted outcome benchmarking has been routinely reported in the annual report of the trauma registry since 2003 [17-21]. Quite recently Osler at al. [22] published a new trauma mortality prediction model based on the anatomic injury scale. This precise score has been developed on 702,229 patients derived from the national trauma data bank of the American College of Surgeons, Committee on Trauma.

All these scores are static as they can be calculated at one (late) time. The TS and the RTS can be used before the patient is admitted to hospital. The more complex scores (ISS, TRISS, ICISS, NISS, ASCOT, Rixen score, RISC score, and the TMPM-score by Osler) can be calculated usually during early intensive care unit (ICU) stay after all injuries have been identified and classified. This is sufficient for many potential applications but a prognosis cannot be provided by the scores during the early period of resuscitation.

The primary aim of this study was to develop a sequential, dynamic score that allows a prognosis at several early stages based on the information that is available at a particular time. The precision of the prognosis should increase step by step because of the increasing additional information. Based on easily available routine data the medical team should be aided by a simple and useful tool to identify patients at high risk and to predict the prognosis of an individual major trauma patient very early.

A secondary aim was to analyse the explanatory power and the impact of the different segments inves- tigated (patients basic data, prehospital phase, and early and late trauma room phase) to be able to assess the relevance of the sectors.

We hypothesized that it is possible to develop, evaluate, and validate such a score based on the analysis of the data of the Trauma Registry of the German Trauma Society.

\section{Methods \\ Data Collection}

The Trauma Registry of the German Trauma Society (DGU) was initiated in 1993 by the society's Working Group on Polytrauma to collect data on patients with polytrauma within German-speaking countries (Germany, Austria, and Switzerland). This trauma registry is a prospective, multicenter, standardized, and anonymized, documentation of severely injured patients. Basic facts about the patient $(\mathrm{P})$, prehospital (A), and treatment in the trauma room (B), and the subsequent course in the ICU $(\mathrm{C})$, and scoring and outcome data (D) are entered continuously to a webbased data server. Every injured patient admitted to one of the participating trauma hospitals*** with an injury severity score (ISS) of 16 or more, or ICU treatment is documented. Data are submitted to a central database at the Institute for Research in Operative Medicine (IFOM) at the University of Witten/ Herdecke in Cologne. Irreversible anonymity of data is guaranteed both for the individual patient and the participating hospital, so the registry comprises epidemiologic, physiologic, laboratory, diagnostic, operative, interventional, and intensive care medical data as well as scoring and outcome data [23].

We analyzed the database for 2002-2006 that contained 17,935 patients. Exclusion criteria were ISS $<9$ (minus 2,424 patients), and patients who were transferred from other hospitals (minus 2,963 patients). After the exclusion criteria had been applied 12,548 patients remained for analysis.

\section{StATISTICAL ANALYSIS}

Retrospective statistical analysis was performed in several steps.

1. The data were screened to identify different, potentially appropriate variables with prognostic power towards the target variable mortality (descriptive and bivariate analysis after dichotomization, $\chi^{2}$ test was used (without continuity correction), cut off p-value $<0,05)$. Survival was defined as survival to discharge. According to the structure of the Trauma Registry the different segments for the score have been defined as basic patient data $(\mathrm{P})$, prehospital phase (A), early trauma room phase (B1) and late trauma room phase (B2).

The patients basic data $P$ (dichotomized) comprised age $\geq 60$ vs. $<60$ years, male vs. female sex, and presence vs. no presence of pre-existing diseases.

The prehospital parameter A (dichotomized) comprised trauma mechanism (blunt vs. penetrating), intubation vs. no intubation, peripheral oxygen saturation ob- 
tained by pulse oximetry $\left(\mathrm{SpO}_{2}\right) \geq 90$ vs. $<90 \%$, insertion vs. no insertion of a chest tube, systolic blood pressure $\geq 90$ vs. $<90 \mathrm{mmHg}$, pulse rate $\geq 120$ vs. $<$ $120 / \mathrm{min}$, closed chest cardiac massage (CCCM) vs. no CCCM, amount of volume substitution $\geq 2000$ vs. $<$ $2000 \mathrm{~mL}, \mathrm{GCS}>8$ vs. $\leq 8$ and anisocoria vs. no anisocoria, all on-scene. Anisocoria was defined as pupillary inequality or both pupils dilated and fixed to light (pupillary dysfunction).

The early trauma room phase parameter B1 (dichotomized) comprised oxygen saturation $\left(\mathrm{SpO}_{2}\right) \geq 90$ vs. $<90 \%$, horowitz coefficient $\left(\mathrm{paO}_{2} / \mathrm{FiO}_{2}\right) \geq 300$ vs. $<300$, systolic blood pressure $\geq 90$ vs. $<90 \mathrm{mmHg}$, pulse rate $\geq 120$ vs. $<120 / \mathrm{min}$, haemoglobin concentration $>8$ vs. $\leq 8 \mathrm{~g} / \mathrm{dL}$, base excess $>-8$ vs. $\leq-8$, thromboplastin time $\geq 50$ vs. $<50 \%$, GCS $>8$ vs. $\leq 8$, anisocoria vs. no anisocoria and temperature $\geq 35$ vs. $<$ $35^{\circ} \mathrm{C}$, all on admission to the trauma room.

The late trauma room phase parameter B2 (dichotomized) comprised ISS $\geq 16$ vs. $<16$, ISS $\geq 25$ vs. $<25$, maximum of the AIS (4 or 5) vs. AIS less than maximum (1-3), AIS $_{\text {head }} \geq 3$ vs. $<3$, AIS $_{\text {thorax }} \geq 3$ vs. $<3$, AIS $_{\text {abdomen }} \geq 3$ vs. $<3$, AIS $_{\text {extremities }} \geq 3$ vs. $<3$, CCCM vs. no CCCM, need vs. no need for blood transfusion, need vs. no need for massive blood transfusion $(\geq 10$ units of packed red blood cells) and need vs. no need for emergency operation (interruption of trauma room diagnostics), all during the treatment in the trauma room until transfer to the ICU or operating theatre (OR).

2. After the screening only those patients with complete data for all mentioned, significant variables were analysed (descriptive and bivariate analysis, same dichotomization, target variable $=$ mortality; $\chi^{2}$ test). Variables with high rates of missings have been excluded.

3. The data were randomized (ratio of $3: 1$ ) to obtain a development and a validation data set. The data sets were compared to detect potential differences using Student's t-test or $\chi^{2}$ test as appropriate (both two sided).

4. The obtained variables were included into logistic regression models with the target variable being mortality (stepwise forward) as follows: model P, A, P+A, $\mathrm{B} 1, \mathrm{P}+\mathrm{A}+\mathrm{B} 1, \mathrm{~B} 2$, and final model $\mathrm{P}+\mathrm{A}+\mathrm{B} 1+\mathrm{B} 2$.

5. Score quality parameters were calculated as discrimination parameters (prognosis of survivors and nonsurvivors, difference between survivors and non-survivors, sensitivity, specificity, overall accuracy, Nagelkerkes $\mathrm{R}^{2}$ [24], area under the curve (AUC) of the receiver operating characteristic (ROC)), precision parameters (observed vs. predicted score mortality) and calibration parameters (Goodness of fit statistics according to Hosmer-Lemeshow [25]). As a reference the quality parameters have also been calculated for the TRISS and the RISC score.

6. In a further step the explanatory power was calculated, so that we were able to assess the proportion of the predictive power of the different segments that can be explained by the model rather than by variation. This is reflected by the Nagelkerkes $\mathrm{R}^{2}$ of the named segments [24]. When the $\mathrm{R}^{2}$ of the full logistic regression model $(\mathrm{P}+\mathrm{A}+\mathrm{B} 1+\mathrm{B} 2)$ minus the variables of model $\mathrm{P}$ is calculated, the difference $(\Delta)$ of $\mathrm{R}^{2}$ from the full model can be calculated. Done in a similar way for the other three segments this enables one to calculate the proportion of each in relation to the sum of all four $\Delta \mathrm{s}(=100 \%)$. The explanatory power - a tool for the assessment of the relative impact of every segment to mortality - can be determined [26].

7. In a final step the score formula were integrated into an Excel spreadsheet. The score prognosis of a given patient can therefore be calculated automatically and quite easily on the internet. A spreadsheet for the easy calculation of the sequential trauma score is available at: www.sequential-trauma-score.com

95\% confidence intervals (CI 95\%) have been calculated where appropriate. Statistical significance was assessed at $p<0.05$. Statistical analysis was performed using SPSS Version 15.0 (SPSS Inc., Chicago, IL, USA).

\section{RESULTS}

After the exclusion criteria had been applied 12,548 patients remained for analysis. These patients had a mean age of $42.5 \pm 20.7$ (standard deviation, SD) years, $72.8 \%$ were male, $94.5 \%$ sustained blunt trauma, the mean ISS was $26.0 \pm 13.9$ and the overall mortality rate was $16.5 \%$. Bivariate testing showed that all of the variables were significantly related to mortality $(p<0.001$ in each case, step 1) with the exception of trauma mechanism and AIS $_{\text {thorax }}$ which therefore have been excluded. The missing rates varied from $0 \%$ (age, sex, ISS, AIS) to $6,8 \%$ (GCS), to $12,6 \%$ (pupillary dysfunction), to $20,2 \%$ (thromboplastin time) and up to $59,1 \%$ for the base excess. The variables temperature and Horowitz coefficient (missings of $75,3 \%$ and $81,6 \%$ respectively) have been taken out (step 2 ).

The remaining variables were then operated to the 12,548 patients giving a total of $\mathrm{n}=2,354$ patients with complete data of all variables included $(=18.8 \%$; $2,354 / 12,548)$. The characteristics of these $2,354 \mathrm{pa}-$ tients are listed in Table 1.

Bivariate testing showed that all variables were significantly related to mortality $(\mathrm{p}<0.001$, cut off $\mathrm{p}$-value $<0,05)$ with the exception of $\operatorname{sex}(p=0,24)$, of insertion of a chest tube $(p=0,16)$, prehospital volume substitution $(p=0,64)$ and $\mathrm{AIS}_{\text {abdomen }}(\mathrm{p}=0,66)$ which therefore have been excluded from the logistic regression.

Randomization of the 2,354 patients with a ratio of 3:1 led to a development data set with 1,760 patients and a validation data set of 594 patients. There were no significant differences between the two data sets when they were compared ( $p$-values $>0.05$ in each case, step 3).

The variables significantly associated with survival in the bivariate analysis were then entered into several logistic regression models as shown in Table 2 (step 4). Model P (patients basic data) could be started with 2 variables (age and pre-existing diseases), model A 
Table 1. Characteristics of the 2,354 major trauma patients with complete data of all included variables.

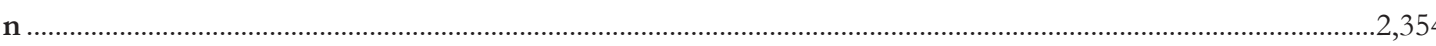

Characteristic

Mean $( \pm \mathrm{SD})$ or No $(\%)$

Age (years)

\section{Basic patient data $(\mathbf{P})$}

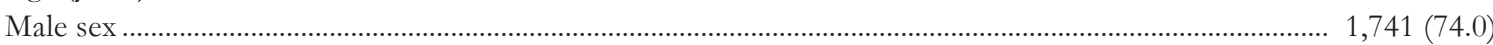

Pre-existing disease

\section{Prehospital (A)}

Blunt trauma .

Intubation rate.

Peripheral oxygen saturation $(\mathrm{SpO} 2, \%)$.

Chest tube insertion rate....

Systolic blood pressure $(\mathrm{mmHg})$

Pulse rate (beats/min)....

CCCM rate.

Amount of volume substitution (mL)

GCS on-scene (points)

Rate of anisocoria

Trauma Room / In hospital (B)

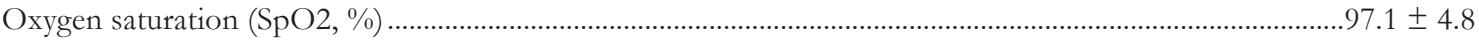

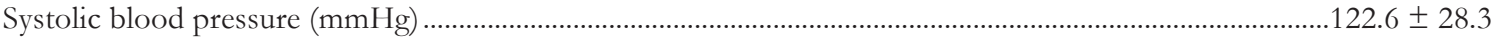

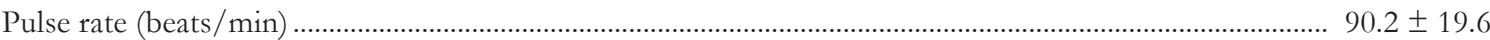

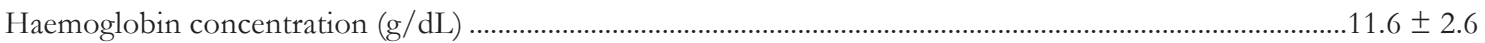

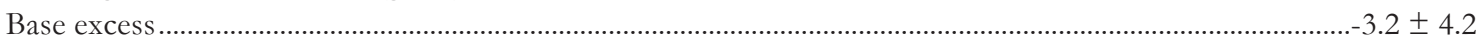

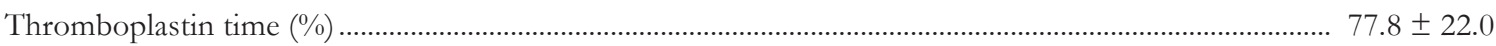

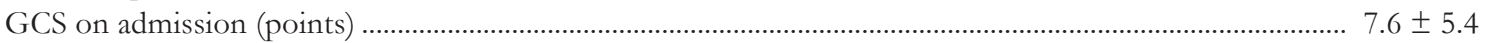

Rate of anisocoria ................................................................................................................................ $319(13.6)$

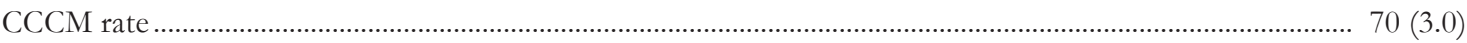

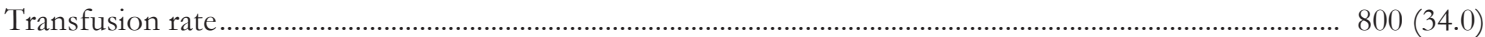

Rate of massive blood transfusion until ICU $(\geq 10$ PRBC) ............................................................................ 208 (8.8)

Emergency operation rate (interruption of trauma room diagnostics) ......................................................... 136 (5.8)

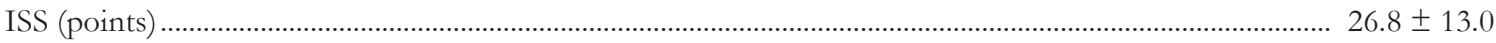

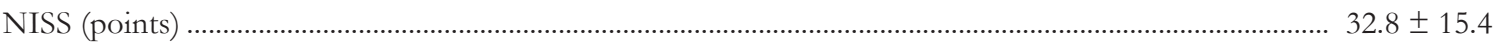

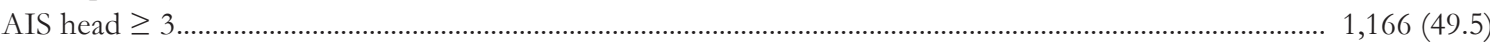

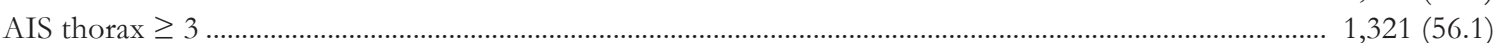

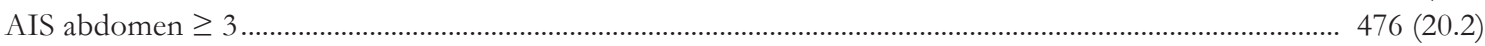

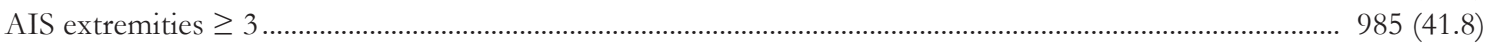

Overall mortality rate ........................................................................................................................... $305(13.0)$

CCCM closed chest cardiac massage; GCS Glasgow Coma Scale; ICU Intensive care unit; PRBC packed red blood cells; ISS Injury severity score; NISS New injury severity score; AIS abbreviated injury scale

(prehospital phase) with 7 variables (intubation, oxygen saturation, blood pressure, pulse rate, CCCM, GCS, and anisocoria), model B1 (early trauma room phase) with 8 variables (oxygen saturation, blood pressure, pulse rate, haemoglobin level, base excess, thromboplastin time, GCS, and anisocoria) and model B2 (late trauma room phase) with 8 variables (CCCM, blood transfusion, massive transfusion, emergency operation, ISS $\geq 16$, ISS $\geq 25$, AIS $_{\text {head }}$, and AIS $\left._{\text {max }}\right)$. The

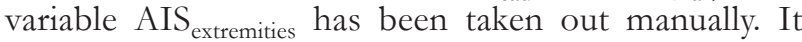
seems that a high AIS of the extremities is rather protective than predictive of mortality because limb injuries are in most cases not life-threatening. Table 2 shows the relevant variables that were included by the respective models for each segment. Nine variables have been included in the final model in which one variable is from segment $\mathrm{P}$ (age), two from the prehospital phase A (GCS and anisocoria), three from the early trauma room phase B1 (base excess, thromboplastin time and anisocoria) and three from the late trauma room phase B2 (CCCM, massive transfusion and $\mathrm{AIS}_{\max }=4$ or 5 ). The score calculation formulas are shown in the appendix.

Tables 3 and 4 show the quality parameters of the different models (development and validation data set). The models are increasingly able to discriminate between survivors and non-survivors, the specificity is high, the AUC of the ROC of 0.90 (95\% CI 0.88-0.92, final model $\mathrm{P}+\mathrm{A}+\mathrm{B} 1+\mathrm{B} 2)$ is significantly higher than that of TRISS and comparable to that of RISC. The ROCs of the different models are shown in Figure 1 and 2 . The precision parameters indicate that observed 
Table 2. Logistic Regression models $\mathrm{P}, \mathrm{A}, \mathrm{P}+\mathrm{A}, \mathrm{B} 1, \mathrm{P}+\mathrm{A}+\mathrm{B} 1, \mathrm{~B} 2$, and $\mathrm{P}+\mathrm{A}+\mathrm{B} 1+\mathrm{B} 2$.

\begin{tabular}{|c|c|c|c|c|}
\hline Variable & Regression coefficient $\beta$ & $\mathrm{p}$ value & Odds ratio $\left(\mathrm{e}^{\beta}\right)$ & CI 95\% \\
\hline \multicolumn{5}{|l|}{ Model P (Patients basic data) } \\
\hline Age $\geq 60$ & 1.234 & $<0.001$ & 3.44 & $2.57-4.60$ \\
\hline Constant & -2.268 & $<0.001$ & - & - \\
\hline \multicolumn{5}{|l|}{ Model A (Prehospital phase) } \\
\hline Blood pressure $<90 \mathrm{mmHg}$ & 0.891 & $<0.001$ & 2.44 & $1.66-3.59$ \\
\hline Pulse rate $\geq 120 / \mathrm{min}$ & 0.554 & 0.018 & 1.74 & $1.10-2.76$ \\
\hline GCS $\leq 8$ on-scene & 1.228 & $<0.001$ & 3.41 & $2.47-4.72$ \\
\hline Anisocoria & 1.164 & $<0.001$ & 3.20 & $2.28-4.51$ \\
\hline Constant & -2.911 & $<0.001$ & - & - \\
\hline \multicolumn{5}{|l|}{ Model P + A } \\
\hline Age $\geq 60$ & 1.653 & $<0.001$ & 5.23 & $3.72-7.34$ \\
\hline Blood pressure $<90 \mathrm{mmHg}$ & 0.983 & $<0.001$ & 2.67 & $1.79-3.99$ \\
\hline Pulse rate $\geq 120 / \mathrm{min}$ & 0,780 & 0.001 & 2.18 & $1.35-3.53$ \\
\hline GCS $\leq 8$ on-scene & 1.353 & $<0.001$ & 3.87 & $2.75-5.44$ \\
\hline Anisocoria & 1.311 & $<0.001$ & 3.71 & $2.59-5.32$ \\
\hline Constant & -3.566 & $<0.001$ & - & - \\
\hline \multicolumn{5}{|l|}{ Model B1 (early trauma room phase) } \\
\hline Oxygen saturation $<90 \%$ & 0.748 & 0.011 & 2.11 & $1.18-3.77$ \\
\hline Base excess $>-8$ & 1.306 & $<0.001$ & 3.69 & $2.47-5.23$ \\
\hline Thromboplastin time $<50 \%$ & 0.997 & $<0.001$ & 2.71 & $1.85-3.97$ \\
\hline GCS $\leq 8$ on trauma room admission & 0.730 & $<0.001$ & 2.08 & $1.40-3.08$ \\
\hline Anisocoria during trauma room phase & 1.907 & $<0.001$ & 6.73 & $4.75-9.53$ \\
\hline Constant & -3.289 & $<0.001$ & - & - \\
\hline \multicolumn{5}{|l|}{ Model P + A + B1 } \\
\hline Age $\geq 60$ & 1.663 & $<0.001$ & 5.28 & $3.69-7.54$ \\
\hline Blood pressure $<90 \mathrm{mmHg}$ on-scene & 0.602 & 0.008 & 1.83 & $1.17-2.85$ \\
\hline GCS $\leq 8$ on-scene & 1.110 & $<0.001$ & 3.03 & $2.10-4.38$ \\
\hline Anisocoria on-scene & 0.700 & 0.004 & 2.01 & $1.25-3.25$ \\
\hline Oxygen saturation $<90 \%$ in trauma room & 0.756 & 0.014 & 2.13 & $1.17-3.89$ \\
\hline Base excess $\leq-8$ & 1.316 & $<0.001$ & 3.73 & $2.40-5.80$ \\
\hline Thromboplastin time $<50 \%$ & 0.947 & $<0.001$ & 2.58 & $1.72-3.90$ \\
\hline Anisocoria during trauma room phase & 1.294 & $<0.001$ & 3.65 & $2.25-5.91$ \\
\hline Constant & -3.901 & $<0.001$ & - & - \\
\hline \multicolumn{5}{|l|}{ Model B2 (late trauma room phase) } \\
\hline $\mathrm{CCCM}$ in trauma room & 1.074 & 0.003 & 2.93 & $1.45-5.90$ \\
\hline Blood transfusion & 0.432 & 0.018 & 1.54 & $1.01-2.21$ \\
\hline Massive transfusion ( $\geq 10$ PRBC) & 0.850 & 0.001 & 2.34 & $1.41-3.86$ \\
\hline AIShead $\geq 3$ & 0.987 & $<0.001$ & 2.68 & $1.84-3.92$ \\
\hline $\mathrm{AIS}_{\max }$ & - & $<0.001$ & - & - \\
\hline $\mathrm{AIS}_{\max }=4$, or: & 0.549 & 0.048 & 1.73 & $1.00-3.28$ \\
\hline $\mathrm{AIS}_{\max }=5$ & 2.483 & $<0.001$ & 12.00 & 6.63-21.64 \\
\hline Constant & -4.235 & $<0.001$ & - & - \\
\hline \multicolumn{5}{|l|}{ Model P + A + B1 + B2 (final model) } \\
\hline Age $\geq 60$ & 1.730 & $<0.001$ & 5.64 & $3.81-8.36$ \\
\hline GCS $\leq 8$ on-scene & 0.752 & $<0.001$ & 2.12 & $1.42-3.20$ \\
\hline Anisocoria on-scene & 0.647 & 0.018 & 1.91 & $1.12-3.28$ \\
\hline CCCM in trauma room & 1.333 & 0.001 & 3.80 & $1.67-8.63$ \\
\hline Massive transfusion ( $\geq 10$ PRBC) & 0.772 & 0.006 & 2.17 & $1.25-3.75$ \\
\hline Base excess $>-8$ & 0.980 & $<0.001$ & 2.66 & $1.64-4.31$ \\
\hline Thromboplastin time $<50 \%$ & 0.711 & 0.003 & 2.04 & $1.28-3.23$ \\
\hline Anisocoria during trauma room phase & 1.251 & $<0.001$ & 3.45 & $2.03-6.01$ \\
\hline $\mathrm{AIS}_{\max }$ & - & $<0.001$ & - & - \\
\hline $\mathrm{AIS}_{\max }=4$, or: & 0.345 & 0.280 & 1.41 & $0.94-2.73$ \\
\hline $\mathrm{AIS}_{\max }=5$ & 2.199 & $<0.001$ & 9.02 & $4.92-16.51$ \\
\hline Constant & -4.857 & $<0.001$ & - & - \\
\hline
\end{tabular}

P Patients basic data; A Prehospital phase; B1 early trauma room phase; B2 late trauma room phase; GCS Glasgow outcome scale; CCCM closed chest cardiac massage; PRBC packed red blood cells; AIS abbreviated injury scale; Regression coefficient and ORs for the variable $\mathrm{AIS}_{\max }$ are not applicable due to the fact that this is a dummy variable. $\mathrm{n}=2,354$ patients, logistic regression (stepwise forward) with target variable mortality; variables sorted according to ATLS ${ }^{\circ}$ A,B,C,D,E-criteria. 
Table 3. Quality parameter of the different models $(n=1,760$, development data set).

\begin{tabular}{|c|c|c|c|c|c|c|c|c|c|}
\hline Model & $\mathbf{P}$ & $\mathbf{A}$ & $\mathbf{P}+\mathbf{A}$ & B1 & $\mathbf{P}+\mathbf{A}+\mathbf{B} 1$ & B2 & $\mathbf{P}+\mathbf{A}+\mathbf{B} 1+\mathbf{B} 2$ & 2 TRISS & RISC \\
\hline \multicolumn{10}{|l|}{ Quality criteria } \\
\hline \multicolumn{10}{|l|}{ Discrimination } \\
\hline Prognosis survivors (S) & 12.5 & 11.1 & 10.3 & 10.3 & 9.2 & 9.6 & 8 & 13.4 & 10 \\
\hline $\begin{array}{l}\text { Prognosis Non-survivors } \\
(\mathrm{N}-\mathrm{S})\end{array}$ & 16.8 & 25.9 & 31.2 & 31.2 & 38.7 & 33.8 & 46.9 & 49 & 48.8 \\
\hline $\begin{array}{l}\text { Difference between S } \\
\text { and N-S }\end{array}$ & 4.3 & 14.8 & 20.9 & 20.9 & 29.5 & 24.2 & 38.9 & 35.6 & 38.8 \\
\hline Sensitivity & 0 & 13.0 & 23.5 & 25.2 & 34.3 & 18.3 & 40.9 & 47.4 & 43.5 \\
\hline Specificity & 100 & 98.4 & 97.8 & 98.9 & 98.1 & 98.3 & 98.2 & 91.6 & 96.2 \\
\hline Overall accuracy & 86.9 & 90.0 & 88.1 & 89.3 & 89.8 & 87.8 & 90.7 & 86.0 & 89.3 \\
\hline Nagelkerkes R ${ }^{2}$ [24] & 0.07 & 0.21 & 0.29 & 0.27 & 0.38 & 0.34 & 0.49 & n.a. & n.a. \\
\hline AUC of ROC & 0.63 & 0.76 & 0.82 & 0.78 & 0.85 & 0.84 & 0.90 & 0.85 & 0.90 \\
\hline $95 \% \mathrm{CI}$ & $0.59-0.67$ & $0.72-0.79$ & $0.79-0.85$ & $0.75-0.82$ & $0.82-0.88$ & $0.81-0.87$ & $0.88-0.92$ & $0.82-0.88$ & $0.89-0.92$ \\
\hline \multicolumn{10}{|l|}{ Precision } \\
\hline Observed mortality (\%) & 13.1 & 13.1 & 13.1 & 13.1 & 13.1 & 13.1 & 13.1 & 13.1 & 13.1 \\
\hline Score prognosis $(\%)$ & 13.1 & 13.1 & 13.1 & 13.1 & 13.1 & 13.1 & 13.1 & 18.0 & 15.1 \\
\hline \multicolumn{10}{|l|}{ Calibration } \\
\hline H-L [25] & n.a. & 1.34 & 5.29 & 2.36 & 4.19 & 4.66 & 11.48 & 136.10 & 17.30 \\
\hline $\mathrm{p}$-value & n.a. & 0.511 & 0.259 & 0.500 & 0.522 & 0.701 & 0.120 & $<0.001$ & 0.027 \\
\hline
\end{tabular}

P Patients basic data; A Prehospital phase; B1 early trauma room phase; B2 late trauma room phase; TRISS trauma and injury severity score, RISC revised injury severity classification score; S survivors; N-S non-survivors; AUC area under the curve. ROC receiver operating characteristic; CI confidence interval; H-L Goodness of fit statistics according to Hosmer and Lemeshow [25]; n.a. not applicable

Table 4. Quality parameter of the different models $(n=594$, validation data set).

\begin{tabular}{|c|c|c|c|c|c|c|c|c|c|}
\hline Model & $\mathbf{P}$ & A & $\mathbf{P}+\mathbf{A}$ & B1 & $\mathbf{P}+\mathbf{A}+\mathbf{B} 1$ & B2 & $\mathbf{P}+\mathbf{A}+\mathbf{B} 1+$ & TRISS & RISC \\
\hline \multicolumn{10}{|l|}{ Quality criteria } \\
\hline \multicolumn{10}{|l|}{ Discrimination } \\
\hline Prognosis survivors (S) & 12.5 & 11.5 & 10.7 & 10.7 & 9.6 & 10.5 & 9 & 11.9 & 10 \\
\hline $\begin{array}{l}\text { Prognosis Non-survivors } \\
(\mathrm{N}-\mathrm{S})\end{array}$ & 16.1 & 26.9 & 31 & 31 & 38.1 & 32.2 & 43.5 & 48.8 & 48 \\
\hline $\begin{array}{l}\text { Difference between S } \\
\text { and N-S }\end{array}$ & 3.6 & 15.4 & 20.3 & 20.3 & 28.5 & 21.7 & 34.5 & 36.9 & 38 \\
\hline Sensitivity & 0.0 & 16.0 & 26.7 & 24.0 & 33.3 & 16.0 & 36.0 & 44.6 & 44.0 \\
\hline Specificity & 100.0 & 98.5 & 97.1 & 99.4 & 97.1 & 97.7 & 97.7 & 92.4 & 97.1 \\
\hline Overall accuracy & 87.4 & 88.0 & 88.2 & 89.9 & 89.1 & 87.4 & 89.9 & 87.7 & 90.4 \\
\hline Nagelkerkes R ${ }^{2}$ [24] & - & - & - & - & - & - & - & - & - \\
\hline AUC of ROC & 0.61 & 0.75 & 0.79 & 0.77 & 0.82 & 0.82 & 0.87 & 0.84 & 0.89 \\
\hline $95 \% \mathrm{CI}$ & $0.54-0.68$ & $0.68-0.81$ & $0.73-0.85$ & $0.71-0.83$ & $0.76-0.88$ & $0.77-0.87$ & $0.82-0.91$ & $0.79-0.90$ & $0.85-0.93$ \\
\hline \multicolumn{10}{|l|}{ Precision } \\
\hline Observed mortality (\%) & 12.6 & 12.6 & 12.6 & 12.6 & 12.6 & 12.6 & 12.6 & 12.6 & 12.6 \\
\hline Score prognosis $(\%)$ & 13.0 & 13.4 & 13.3 & 13.3 & 13.2 & 13.3 & 13.4 & 16.5 & 14.8 \\
\hline \multicolumn{10}{|l|}{ Calibration } \\
\hline $\mathrm{H}-\mathrm{L}[25]$ & n.a. & 3.96 & 4.68 & 4.66 & 6.92 & 11.04 & 6.01 & 35.60 & 8.34 \\
\hline p-value & n.a. & 0.138 & 0.322 & 0.200 & 0.230 & 0.137 & 0.420 & $<0.001$ & 0.400 \\
\hline
\end{tabular}

P Patients basic data; A Prehospital phase; B1 early trauma room phase; B2 late trauma room phase; TRISS trauma and injury severity score, RISC revised injury severity classification score; S survivors; N-S non-survivors; AUC area under the curve. ROC receiver operating characteristic; CI confidence interval; H-L Goodness of fit statistics according to Hosmer and Lemeshow [25]; n.a. not applicable

and predicted prognoses are congruent. The calibration parameters show that the models are well calibrat- ed as low Hosmer-Lemeshow-goodness of fit values and non-significant (!) p-values indicate good calibra- 


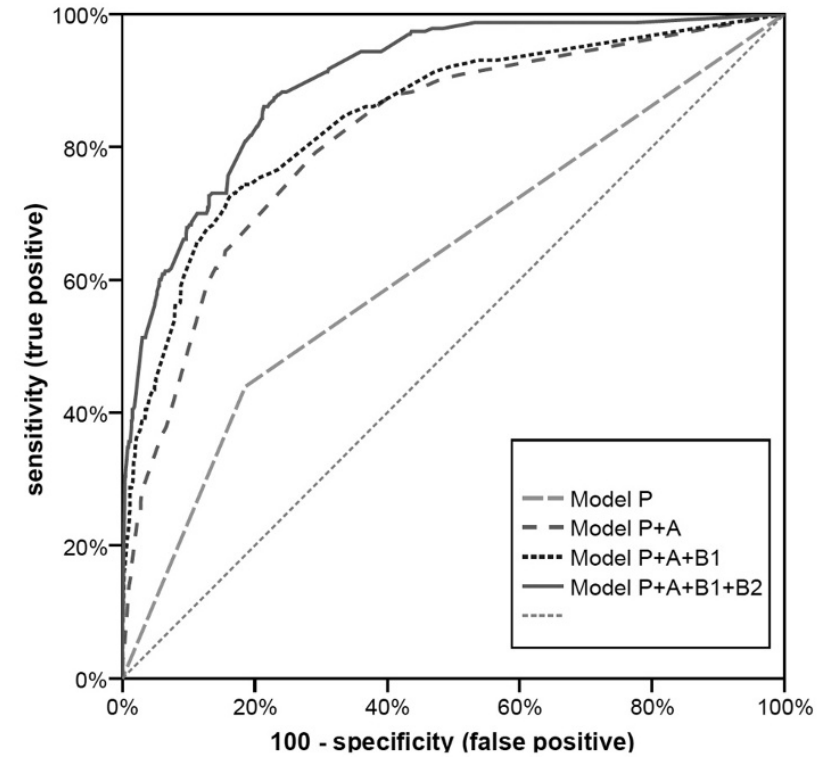

Fig. 1. Receiver operating characteristic curves (ROCs) of the models $\mathrm{P}, \mathrm{P}+\mathrm{A}, \mathrm{P}+\mathrm{A}+\mathrm{B} 1$ and $\mathrm{P}+\mathrm{A}+\mathrm{B} 1+\mathrm{B} 2$ (final model). $\mathrm{P}$ patients basic data, A prehospital phase, B1 early trauma room phase, B2 late trauma room phase.

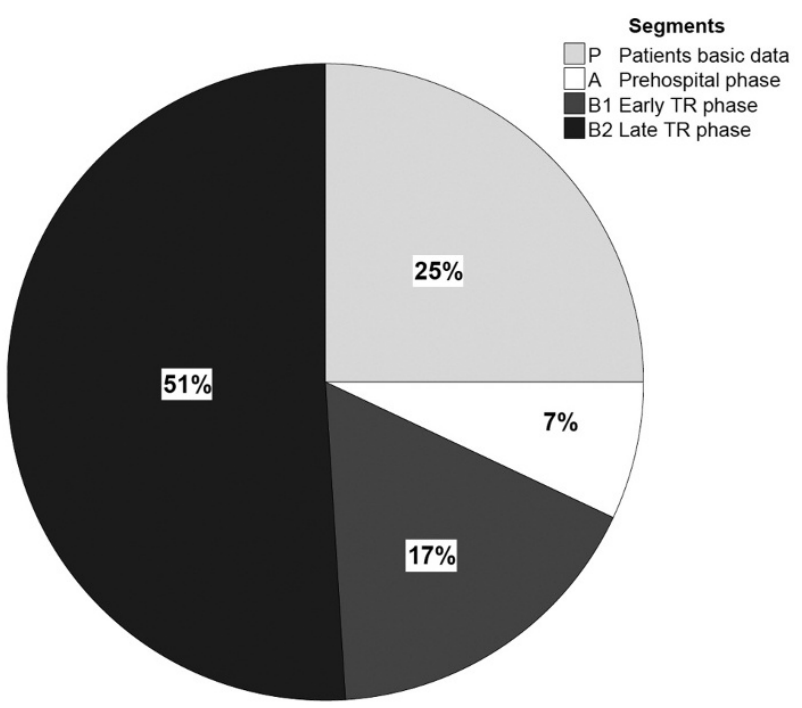

Fig. 3. Relative contribution of the segments A, P, B1 and B2 to mortality explainable by the model (explanatory power).

tion of scores.[2, 4] The same direction of the data is evident for the validation data set (Table 4).

The explanatory power of the four segments was calculated to be $25 \%$ for $\mathrm{P}, 7 \%$ for $\mathrm{A}, 17 \%$ for $\mathrm{B} 1$ and $51 \%$ for $\mathrm{B} 2$, indicating that the trauma room phase $(\mathrm{B} 1+\mathrm{B} 2,68 \%)$ comprises most predictive relevance followed by the patients basic data and the prehospital phase (Fig. 3) (step 6).

Finally the score formulas have been integrated into an Excel spreadsheet. So the probability of survival for a given patient may be calculated quite easily. www.sequential-trauma-score.com

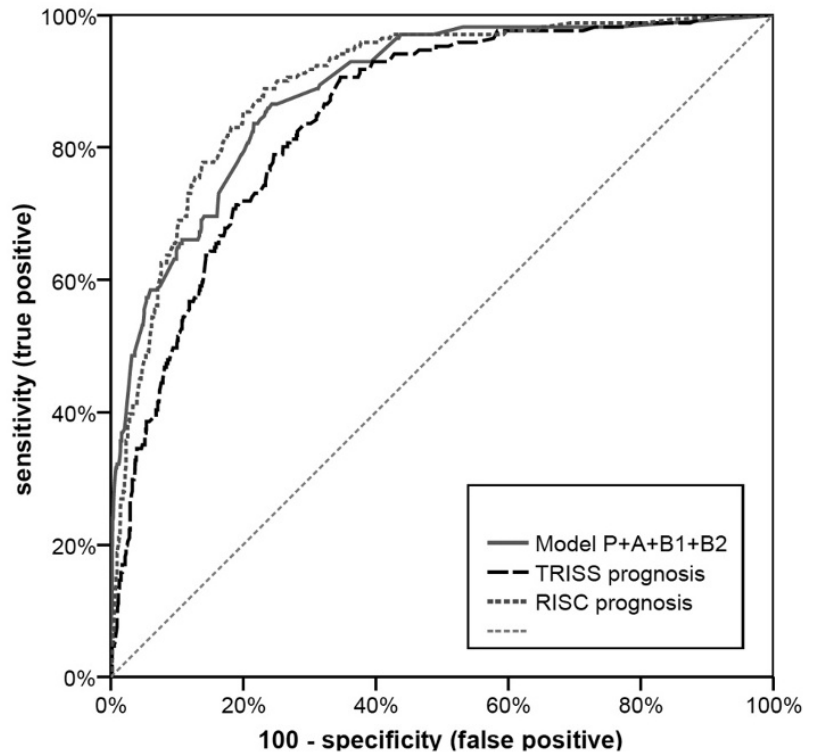

Fig. 2. Receiver operating characteristic curves (ROCs) of the final model $(\mathrm{P}+\mathrm{A}+\mathrm{B} 1+\mathrm{B} 2)$, TRISS and RISC. P patients basic data, A prehospital phase, B1 early trauma room phase, B2 late trauma room phase. TRISS trauma and injury severity score, RISC revised injury severity classification score.

\section{Discussion}

Scoring systems enable the physician to transform complex clinical circumstances into a single number and to translate different severity codes into a common language. The cohort investigated in this study comprises severely and multiply-injured, blunt trauma patients expressed by the high ISS of 26.8 and the NISS of 32.8 respectively. The sequential trauma score (STS) therefore seems to be best suited to patients with "real" major trauma.

In contrast to many other scores the STS comprises the base excess and coagulation variables that have been proved to contain relevant predictive power [1416, 19]. The score integrates the predictive power of the oxygen saturation obtained by pulse oximetry on admission to the trauma room and pathologic pupillary function on-scene as well as on admission which is indicated by the high odds ratios (OR) in the respective logistic regression models. Peripheral oxygen saturation has never before been used to predict mortality. In 2006 Raux et al. [27] reported that $\mathrm{SpO}_{2}$ did not add a statistically significant value to other variables to predict trauma mortality in 675 patients which is in contrast to our findings. To the best of our knowledge pupillary function has not previously been evaluated or integrated into major trauma scoring systems. However, in neurosurgery pupillary dysfunction is regarded as an important predictor for the outcome of patients with traumatic brain injury [28-31]. The high rate of severe traumatic brain injuries $\left(\right.$ AIS $_{\text {head }} \geq 3$ ) of about $50 \%$ in our cohort probably strengthens the predictive effect of the variable "pupillary dysfunction".

The sequential trauma score is based on results from 2,354 patients. Other commonly used scores are clearly based on more patients. For example, the trau- 
ma mortality prediction model by Osler [22] is based on 702,229 trauma victims derived from the national trauma data bank. However, the quality criteria of the STS indicate good discrimination, high specificity $(98.2 \%)$, high overall accuracy $(90.7 \%)$, and a high AUC of the ROC (0.90) as well as excellent precision and calibration. The STS has better quality criteria than the well-known TRISS and RISC when applied to the investigated cohort. When other well-known scores are applied to the Trauma Registry of the German Trauma Society $(1993-2000, \mathrm{n}=8056)$ the AUC of the ROCs are at the highest for the Score by Rixen (AUC $=0.88,95 \%$ CI 0.85-0.90), followed by the ASCOT (AUC $=0.85,95 \%$ CI 0.82-0.88), the NISS $(\mathrm{AUC}=0.80,95 \%$ CI $0.77-0.84)$, the ISS (AUC = $0.79,95 \%$ CI $0.75-0.82$ ) and the RTS (AUC $=0.77$, 95\% CI 0.73-0.80) [19]. This emphasizes that the STS is a simple and improved score compared with these.

The TMPM-score recently introduced by Osler [22] shows an AUC of the ROC of 0.93 indicating a very high discrimination quality, but the score comprises injury severity, age, gender and mechanism of injury, and no other variables. The National Trauma Data Bank comprises about $75 \%$ patients with an ISS of $<16$ [32]. The mean ISS within the Trauma Registry is 24.5 containing $74 \%$ patients with an ISS of greater than 16 [33]. The two collectives (minor and major trauma) are therefore comparable only with limitations. With its high overall accuracy and the AUC of the ROC of 0.90 the STS is one of the most precise trauma scores.

To the best of our knowledge the STS is the first sequential, dynamic score that takes the information available at the different described points in time (patients' basic data, prehospital phase, early and late trauma room phase). Its precision increases with every additional piece of information available later on. If they know the four relevant predictors from the prehospital segment - blood pressure, pulse rate, GCS and pupillary function - the medical team at the scene can estimate the first assessment of prognosis. On admission to the trauma room the peripheral oxygen saturation and the pupillary function can easily be recorded within a structured primary survey according to the ATLS ${ }^{\circledR}$ guidelines [34]. The base excess can be determined from the first blood gas analysis and the thromboplastin time should be available about 25 minutes after trauma room admission. After the completion of the diagnostic phase (sonography, X-ray and or $\mathrm{CT}$ /whole body $\mathrm{CT}$ ) in the trauma room it is obvious whether the patient has a severe injury with a maximum AIS code (either AIS $=4$ or 5 ). At this point in time we need to know only whether the patient has an AIS 4 or 5 injury. We can then make a reliable prediction of the expected probability of survival before the patient is transferred to the ICU. So the Sequential Trauma Score might help the trauma team to identify critically injured high risk patients earlier. The STS can be seen as a tool to quantify the surgeons "gut feeling" which could be an advantage especially for young, not so experienced trauma team members. The STS could also guide the decision which patient damage control surgery should be applied to. This issue should be evaluated in further studies. We assume that below a predicted probability of survival of $75 \%$ the principles of damage control surgery should at least be considered. To make the STS calculation easy and practicable a spreadsheet has been designed, which is available online (see results).

In case of an early death of a trauma patient the STS could be used to estimate whether the death might have been preventable or rather not. This might also help the medical team as a tool for "exculpation".

To our opinion 7 of the 11 STS variables can principally be addressed by an appropriate therapy. For example it should be tried to elevate the blood pressure and reduce tachycardia by differentiated volume therapy and of course surgical control of the bleeding. Low GCS and pupillary dysfunction should be addressed by correct perfusion pressures for the brain, intracranial pressure therapy and by surgical evacuation of intracranial hematomas, if indicated. Low oxygen saturation should be addressed by optimal airway management and ventilation. Low base excess as an indicator for suboptimal tissue perfusion should be addressed by an aggressive anti-shock therapy (volume, transfusion, surgical control of the bleeding). Low thromboplastin time should be addressed by surgical control of the bleeding, rapid transfusion and optimal coagulatory management. So the STS not only comprises prognostic information but also indicates the most relevant therapeutic targets.

A secondary aim of this study was to calculate the explanatory power of each segment: these were $25 \%$ for the patients basic data, 7\% prehospital, 17\% for early trauma room phase and $51 \%$ for late trauma room phase. The patient's basic data are fixed and not accessible for medical treatment. The small contribution of $7 \%$ of the prehospital phase is surprising. This emphasizes that it is quite difficult on-scene to make a correct assessment of the patients "real" condition. With a total of $68 \%$ the trauma room phase $(\mathrm{B} 1+\mathrm{B} 2)$ seems to have the most impact on mortality. This underlines the importance of correct triage of patients with major trauma to be able to transport them to adequate facilities with a structured and routine trauma protocol. These calculations are a tool to assess the dimension of each segment knowing that they indicate results of a mathematical model. To the best of our knowledge similar calculations have not yet been made in trauma management. Comparable calculations in intensive care medicine have been done by Zimmermann et al. [26].

There are several limitations to our study. Because some data were missing from the Trauma Registry the sequential trauma score could be developed on only 2,354 patients. This might bias the results. Because the variable "hypothermia" was rarely recorded it had to be excluded, although its high predictive power has been proved within our and other trauma registries $[35,36]$. We decided to cope the problem with the missings by considering only "complete cases" which is necessary for the final multivariate regression model. We declined to use imputation of missing values because this procedure could also lead to a systematic bias.

The variable $\mathrm{AIS}_{\max }$ in the final model is divided into $\mathrm{AIS}_{\max }=4$ or $\mathrm{AIS}_{\max }=5$. The $\mathrm{AIS}_{\max }=4 \mathrm{com}-$ 
ponent is the only variable that is not significant in the final model. Because it is appropriate to be able to choose between $\mathrm{AIS}_{\max }=4$ or $\mathrm{AIS}_{\max }=5$, and not every severely-injured patient has an injury with an AIS of 5, we left this variable in the final model knowing that it did not add much additional predictive power.

96.2 of the cohort investigated sustained blunt trauma. Thus the STS should not be applied to patients with penetrating trauma.

There is also potentially different intercenter consistency in grading injuries (AIS, ISS). Another limiting factor is the relatively small size of our validation group. Furthermore the generalizability of our results has to be seen critically due to unquantifiable differences of the health care systems in different countries. To address the mentioned last two issues a prospective and external validation of the sequential trauma score outside the Trauma Registry of the German Trauma Society is necessary to verify our findings. This should be done in the future.

In conclusion, the sequential trauma score developed in this study is the first sequential score that provides a prognosis of patients with blunt major trauma at several points in time. With every additional piece of information the precision increases. Based on easy available routine data the medical team has a simple, useful tool to identify patients at high risk and to predict the prognosis of an individual patient with major trauma very early, quickly and precisely.

Statements:

This study has the full approval of the ethics committee of the Ludwig-Maximilians-University of Munich (LMU), Germany.

There are no conflicts of interest.

There was no funding for this particular study, however, the Trauma Registry of the German Trauma Society (Deutsche Gesellschaft für Unfallchirurgie, DGU) in general was partly funded by the Deutsche Forschungsgemeinschaft (DFG) Ne 385 / 5 and by a grant of Novo Nordisk A/S, Bagsvaerd, Denmark.

Dr Huber-Wagner declares: I had full access to all the data in the study and had final responsibility for the decision to submit for publication.

\section{Acknowledgements: \\ ** Working group on Polytrauma - German Trauma Society (DGU)}

Sektion Notfall-, Intensivmedizin und Schwerverletztenversorgung - NIS - der DGU

Chairmen: A. Seekamp, PhD and S. Ruchholtz, PhD; chairmen of working subgroup "Trauma Registry": R. Lefering, $\mathrm{PhD}$ and Th. Paffrath, MD.)

*** Participating hospitals (alphabetic)

Universitätsklinik der RWTH Aachen; Zentralklinikum Augsburg; Kreiskrankenhaus Bad Hersfeld; Charité - Campus Virchow-Klinikum Berlin; Martin-Luther-Krankenhaus Berlin; Klinikum Berlin-Buch; BG-Unfallklinik BerlinMahrzahn; Krankenanstalten Gilead Bielefeld; BG-Klinik Bochum Bergmannsheil; Knappschaftskrankenhaus der Ruhr-Universität Bochum; Friedrich-Wilhelms-Universität Bonn; Zentralkrankenhaus Sankt-Jürgen-Straße Bremen; Zentralkrankenhaus Bremen Ost; Klinikum BremerhavenReinkenheide; Allgemeines Krankenhaus Celle; Klinikum Chemnitz; Klinikum Dessau; Klinikum Lippe-Detmold;
Krankenhaus Dresden-Neustadt; Technische Universität Dresden; Krankenhaus Dresden-Friedrichstadt; HeinrichHeine-Universität Düsseldorf; Klinikum Erfurt; Kreiskrankenhaus Eschwege; Universitätsklinikum Essen; Evang. Krankenhaus Lutherhaus Essen; BG Unfallklinik Frankfurt / Main; Universitätsklinik Frankfurt/Main; Klinikum Frankfurt/Oder; Klinikum Fürth; JohanniterKrankenhaus Geesthacht; Städtisches Klinikum Görlitz; Klinik an Eichert Göppingen; Georg-August-Universität Göttingen; Universität Graz (Österreich); Allg. Unfallvericherungsanstalt Graz (Österreich); Kreiskrankenhaus Grevenbroich; Universitätsklinik Groningen (Niederlande); Kreiskrankenhaus Gummersbach; BG-Unfallkrankenhaus Hamburg; Kreiskrankenhaus Hameln; Medizinische Hochschule Hannover; Krankenhaus Hannover-Nordstadt; Friederikenstift Hannover; Ev. Krankenhaus Hattingen; Orthopäd. Universitätsklinik Heidelberg; St. Bernward Krankenhaus Hildesheim; Universität des Saarlandes Homburg / Saar; Waldviertel Klinikum Horn (Österreich); LKH Judenburg-Knittelfeld (Österreich); Städt. Klinikum Karlsruhe; Christian-Albrechts-Universität Kiel; Chirurgischer Lehrstuhl der Universität zu Köln; Städt. Klinikum KölnMerheim; Allg. öff. Krankenhaus Krems/Donau (Österreich); Städt. Klinikum St. Georg Leipzig; Universität Leipzig; Ev. Krankenhaus Lengerich; Allg. öffentl. Krankenhaus Linz (Österreich); Ev. Krankenhaus Lippstadt; Universitätsklinikum Lübeck; BG Unfallklinik Ludwigshafen; St.-Marien-Hospital Lünen; Krankenhaus Altstadt; Städt. Klinikum Magdeburg; Otto-von-Guericke-Universität Magdeburg; Johannes-Gutenberg-Universität Mainz; Universitätsklinikum Mannheim; Universität Marburg; Klinikum Minden; Krankenhaus Maria Hilf Mönchengladbach; Klinikum der Universität München - Chirurgische Klinik Campus Innenstadt - Ludwig-Maximilians-Universität München/LMU; Städt. Krankenhaus München-Harlaching; Westfälische Wilhelms-Universität Münster; BG-Unfallklinik Murnau; Lukaskrankenhaus der Städt. Kliniken Neuss; Marienhospital Osnabrück; Vogtland Klinikum Plauen; Klinikum Remscheid; Klinikum Rosenheim; Sana-Krankenhaus Rügen; St. Johanns-Spital - Landeskrankenhaus Salzburg (Österr.); Diakonissenkrankenhaus Schwäbisch Hall; Kreiskrankenhaus Soltau; Johanniter-Krankenhaus der Altmark Stendal; Kreiskrankenhaus Traunstein; BG-Unfallklinik Tübingen; Bundeswehrkrankenhaus Ulm; Universitätsklinik Ulm; Klinikum der Stadt Villingen-Schwenningen; Klinikum Weiden / Opfz.; Asklepios Kreiskrankenhaus Weißenfels; Donauspital Wien (Österreich); Ferdinand-SauerbruchKlinikum Wuppertal; Julius-Maximilians-Universität Würzburg; Universitätsspital ETH Zürich (Schweiz); Rettungsstelle Zusmarshausen.

\section{REFERENCES}

1. Kung H-C, Hoyert DL, Xu J, Murphy SL. Deaths: Final Data for 2005. National Vital Statistics Reports of the CDC. 2008;56(10):1-121.

2. Lefering R. Trauma Score Systems for Quality Assessment. Eur J Trauma. 2002;28:52-63.

3. Bouillon B, Lefering R, Vorweg M, Tiling T, Neugebauer E, Troidl H. Trauma score systems: Cologne Validation Study. J Trauma. 1997 Apr;42(4):652-8.

4. Senkowski CK, McKenney MG. Trauma scoring systems: a review. J Am Coll Surg. 1999 Nov;189(5):491-503.

5. Baker SP, O'Neill B, Haddon W, Jr., Long WB. The injury severity score: a method for describing patients with multiple injuries and evaluating emergency care. J Trauma. 1974 Mar;14(3):187-96.

6. Rating the severity of tissue damage. I. The abbreviated scale. Jama. 1971 Jan 11;215(2):277-80. 
7. Champion HR, Sacco WJ, Carnazzo AJ, Copes W, Fouty WJ. Trauma score. Crit Care Med. 1981 Sep;9(9):672-6.

8. Champion HR, Sacco WJ, Copes WS, Gann DS, Gennarelli TA, Flanagan ME. A revision of the Trauma Score. J Trauma. 1989 May;29(5):623-9.

9. Boyd CR, Tolson MA, Copes WS. Evaluating trauma care: the TRISS method. Trauma Score and the Injury Severity Score. J Trauma. 1987 Apr;27(4):370-8.

10. Osler T, Rutledge R, Deis J, Bedrick E. ICISS: an international classification of disease- 9 based injury severity score. J Trauma. 1996 Sep;41(3):380-6; discussion 386-8.

11. Rutledge R, Fakhry S, Baker C, Oller D. Injury severity grading in trauma patients: a simplified technique based upon ICD-9 coding. J Trauma. 1993 Oct;35(4):497-506; discussion 506-7.

12. Osler T, Baker SP, Long W. A modification of the injury severity score that both improves accuracy and simplifies scoring. J Trauma. 1997 Dec; 43(6): 922-5; discussion 925-6.

13. Champion HR, Copes WS, Sacco WJ, Lawnick MM, Bain LW, Gann DS, Gennarelli T, Mackenzie E, Schwaitzberg S. A new characterization of injury severity. J Trauma. 1990 May;30(5):539-45; discussion 545-6.

14. Rixen D, Raum M, Bouillon B, Schlosser LE, Neugebauer E. Predicting the outcome in severe injuries: an analysis of 2069 patients from the trauma register of the German Society of Traumatology (DGU). Unfallchirurg. 2001 Mar;104(3):230-9.

15. Rixen D, Raum M, Bouillon B, Lefering R, Neugebauer E. Base deficit development and its prognostic significance in posttrauma critical illness: an analysis by the trauma registry of the Deutsche Gesellschaft fur unfallchirurgie. Shock. 2001 Feb;15(2):83-9.

16. Kroezen F, Bijlsma TS, Liem MS, Meeuwis JD, Leenen LP. Base deficit-based predictive modeling of outcome in trauma patients admitted to intensive care units in Dutch trauma centers. J Trauma. 2007 Oct;63(4):908-13.

17. Annual Report of the Trauma Registry of the German Trauma Society (DGU). Working Group on Polytrauma German Trauma Society (DGU) 2003.

www.traumaregister.de

18. Ruchholtz S, Lefering R, Paffrath T, Oestern HJ, Neugebauer E, Nast-Kolb D, Pape H-C, Bouillon B. Reduction in Mortality of Severely Injured Patients in Germany. Dtsch Arztebl. 2008;105(13):225-31.

19. Lefering R. Revised Injury Severity Classification (RISC) - Development and validation of a classification system for severely injured patients based on a large trauma registry. [Revised Injury Severity Classification (RISC) - Entwicklung und Validierung eines Schweregrad-Klassifikationssystems für schwerverletzte Patienten zur Anwendung in einem nationalen Traumaregister] (Habilitationsschrift). IFOM - Institut für Forschung in der Operativen Medizin. Cologne: University Witten/Herdecke, Faculty of Medicine, Germany, 2007. pp. 1-131.

20. Huber-Wagner S, Lefering R, Qvick L, Körner M, Kay M, Pfeifer K, Reiser M, Mutschler W, Kanz K. Effect of whole-body CT during trauma resuscitation on survival: a retrospective, multicentre study. Lancet. 2009;373:145561.

21. Lefering R. Development and validation of the Revised Injury Severity Classification (RISC) score for severely injured patients. Eur J Trauma Emerg Surg. 2009;35:437-47

22. Osler T, Glance L, Buzas JS, Mukamel D, Wagner J, Dick A. A trauma mortality prediction model based on the anatomic injury scale. Ann Surg. 2008 Jun;247(6):1041-8.

23. Ruchholtz S. The Trauma Registry of the German Society of Trauma Surgery as a basis for interclinical quality management. A multicenter study of the German Society of Trauma Surgery. Unfallchirurg. 2000 Jan;103(1):30-7.
24. Nagelkerke NJD. A note on a general definition of the coefficient of determination. Biometrika. 1991;78:691-2.

25. Lemeshow S, Hosmer DW. A review of goodness of fit statistics for use in the development of logistic regression models. Am J Epidemiol. 1982;115:92-106.

26. Zimmerman JE, Kramer AA, McNair DS, Malila FM, Shaffer VL. Intensive care unit length of stay: Benchmarking based on Acute Physiology and Chronic Health Evaluation (APACHE) IV. Crit Care Med. 2006 Oct; 34(10):2517-29.

27. Raux M, Thicoipe M, Wiel E, Rancurel E, Savary D, David JS, Berthier F, Ricard-Hibon A, Birgel F, Riou B. Comparison of respiratory rate and peripheral oxygen saturation to assess severity in trauma patients. Intensive Care Med. 2006 Mar;32(3):405-12.

28. Schynoll W, Overton D, Krome R, Wesolowski D, Wang AM, Wilson A, Coffey M. A prospective study to identify high-yield criteria associated with acute intracranial computed tomography findings in head-injured patients. Am J Emerg Med. 1993 Jul;11(4):321-6.

29. Murray GD, Butcher I, McHugh GS, Lu J, Mushkudiani NA, Maas AI, Marmarou A, Steyerberg EW. Multivariable prognostic analysis in traumatic brain injury: results from the IMPACT study. J Neurotrauma. 2007 Feb;24(2): 329-37.

30. Mushkudiani NA, Hukkelhoven CW, Hernandez AV, Murray GD, Choi SC, Maas AI, Steyerberg EW. A systematic review finds methodological improvements necessary for prognostic models in determining traumatic brain injury outcomes. J Clin Epidemiol. 2008 Apr;61(4): 331-43.

31. Hukkelhoven CW, Steyerberg EW, Habbema JD, Farace E, Marmarou A, Murray GD, Marshall LF, Maas AI. Predicting outcome after traumatic brain injury: development and validation of a prognostic score based on admission characteristics. J Neurotrauma. 2005 Oct;22(10):1025-39.

32. National Trauma Data Bank. Annual Report. 2007.

33. Annual Report of the Trauma Registry of the German Trauma Society (DGU). Working Group on Polytrauma German Trauma Society (DGU) 2007.

34. Advanced Trauma Life Support for Doctors - Student Course Manual. 7th Edition ed. Chicago: American College of Surgeons, 2004.

35. Martin RS, Kilgo PD, Miller PR, Hoth JJ, Meredith JW, Chang MC. Injury-associated hypothermia: an analysis of the 2004 National Trauma Data Bank. Shock. 2005 Aug;24(2):114-8.

36. Shafi S, Elliott AC, Gentilello L. Is hypothermia simply a marker of shock and injury severity or an independent risk factor for mortality in trauma patients? Analysis of a large national trauma registry. J Trauma. 2005 Nov;59(5): 1081-5.

Received: November 21, 2009 / Accepted: January 27, 2010

Address for correspondence:

Dr Stefan Huber-Wagner

Munich University Hospital (LMU)

Department of Trauma Surgery - Campus Innenstadt

Nussbaumstr. 20

80336 Munich

Germany

Phone: $+49(0) 89 / 5160-2511$

Fax: +49(0)89/5160-4934

E-mail: stefan.huber@med.uni-muenchen.de 
APPENDIX

Formula model P:

Probability of survival Ps $=1-(1 /(1+\operatorname{EXP}(2.268-1.234 *$ age $\geq 60)))$

Formula model P+A:

Ps $=1-(1 /(1+\operatorname{EXP}(3.566-1.653 *$ age $\geq 60-1.353 * \mathrm{GCS} \leq 8-1.311 *$ anisocoria $-0.983 *$ blood pressure $<90-0.78 *$ pulse rate $\geq 120)))$

\section{Formula model P+A+B1:}

Ps $=1-(1 /(1+\operatorname{EXP}(3.901-1.663 *$ age $\geq 60-0.602 *$ blood pressure $<90-0.7 *$ anisocoria on-scene $1.11 * \mathrm{GCS} \leq 8-1.294 *$ anisocoria in TR $-1.316 *$ base excess $\leq-8-0.756 * \mathrm{SpO} 2<90-0.947 *$ thromboplastin time $<50 \%))$

\section{Formula model $\mathbf{P}+\mathbf{A}+\mathbf{B} 1+\mathbf{B} 2$ :}

Ps $=1-\left(1 /\left(1+\operatorname{EXP}\left(4.857-1.333 * \mathrm{CCCM}-0.772 *\right.\right.\right.$ massive transfusion $-0.345 * \mathrm{AIS}_{\max }=4 *\left(1-\mathrm{AIS}_{\max }=\right.$ $5)-2.199 * \mathrm{AIS}_{\max }=5-1.73 *$ age $\geq 60-0.752 * \mathrm{GCS} \leq 8-0.647 *$ anisocoria on-scene $-1.251 *$ anisocoria in TR $-0.98 *$ base excess $\leq-8-0.711 *$ thromboplastin time $<50 \%)$ )) 